\title{
Generalized Chebyshev Interpolation and Its Application to Automatic Quadrature
}

\author{
By Takemitsu Hasegawa, Tatsuo Torii and Ichizo Ninomiya
}

\begin{abstract}
A generalized Chebyshev interpolation procedure increasing a fixed number of sample points at a time is developed and analyzed. It is incorporated into an efficient automatic quadrature scheme of Clenshaw-Curtis type. Numerical examples indicate that the present method is efficient not only for well-behaved functions but for those with discontinuous low order derivatives by virtue of adequate error estimation as well as saving of sample points.
\end{abstract}

1. Introduction. We propose an extension of the Clenshaw-Curtis method [2] (hereafter abbreviated as CC method) and the Filippi method [4] which are efficient automatic quadrature schemes for well-behaved integrands over a finite range $[-1,1]$.

These methods rely on a sequence of quadrature rule approximations to the integral. Each approximation requires approximately double the number of function values required by the previous one.

Our modification of these methods has the property that each approximation in the sequence requires only $N$ more function values than the previous approximation. Here $N$ is a power of 2, usually taken to be 8 [8].

In general, the CC method, the Filippi method and the modification proposed by us work in the same way. After calculating an approximation, its accuracy is estimated using the final two or three terms of the Chebyshev series. If this accuracy is within the required tolerance, the calculation is terminated, otherwise, it is continued by calculating a further approximation.

Use of our modification may be particularly advantageous when the number of function values required is high. If at any stage it is in fact necessary to use double the number of function values to attain the required accuracy, all methods are likely to do this. But if only a small number of additional function values is really required, our modification will handle this efficiently and avoid the possibly significant and unnecessary expense of doubling the number of function evaluations.

The abscissae used by our method may be defined as follows. We choose the parameter $N$ which is a power of two, usually 8 . We define the sequence

$$
\alpha_{1}=\frac{1}{4}, \quad \alpha_{2 i}=\alpha_{i} / 2, \quad \alpha_{2 i+1}=\alpha_{2 i}+\frac{1}{2}, \quad i=1,2, \ldots,
$$

\footnotetext{
Received April 8, 1981; revised April 26, 1982, October 13, 1982, and April 19, 1983.
}

1980 Mathematics Subject Classification. Primary 65D05, 65D30; Secondary 41A55.

Key words and phrases. Chebyshev interpolation, approximate integration, Clenshaw-Curtis scheme, automatic quadrature. 
and the abscissae (for the integration interval $[-1,1]$ ) are

$$
x_{i}=\cos 2 \pi \alpha_{i} .
$$

The $l$ th quadrature rule approximation $Q^{\prime}(f)$ provides an interpolatory type approximation, based on the abscissae $x_{i} ; i=1,2, \ldots,(l+1) N-1$. Specifically

$$
Q^{\prime}(f)=\int_{-1}^{1} p_{l}(x) d x .
$$

where $p_{l}(x)$ is an interpolatory polynomial of degree $(l+1) N-2$ satisfying

$$
p_{l}\left(x_{i}\right)=f\left(x_{i}\right), \quad i=1,2, \ldots,(l+1) N-1 .
$$

This paper is principally devoted to deriving convenient formulas for calculating $Q^{\prime}(f), l=0,1, \ldots$, recursively. To this end we introduce the auxiliary polynomial

$$
\omega_{m}(x)=2^{m} \prod_{k=1}^{m}\left(x-\cos 2 \pi \alpha_{k}\right), \quad \omega_{0}(x)=1,
$$

and show (see Section 2) that it can be expressed in the form

$$
\omega_{I N-1}(x)=U_{N-1}(x) \omega_{l-1}\left(T_{N}(x)\right),
$$

where $T_{N}(x)$ and $U_{N}(x)$ are the Chebyshev polynomials of the first and second kinds of degree $N$, respectively.

In Section 3 we show that the interpolatory polynomial may be expressed in the form

$$
p_{l}(x)=\sum_{k=1}^{N-1} A_{0, k} U_{k-1}(x)+\sum_{i=1}^{l} \omega_{i-1}\left(T_{N}(x)\right) \sum_{k=0}^{N-1}{ }^{\prime} A_{i, k} T_{k}(x),
$$

where the prime denotes the summation whose first term is halved. The $l$ th quadrature approximation is then

$$
Q^{\prime}(f)=\int_{-1}^{1} p_{l}(x) d x=\sum_{k=1}^{N} A_{0, k} W_{0, k}+\sum_{i=0}^{l} \sum_{k=0}^{N-1} A_{i, k}^{\prime} W_{i, k},
$$

where $A_{i, k}$ and $W_{i, k}$ do not depend on $l$. Because of this fact, the calculation is conveniently arranged recursively. To proceed from the result $Q^{\prime}(f)$ to calculate $Q^{l+1}(f)$ we need merely $A_{l+1, k} W_{l+1, k}, k=1,2, \ldots, N$. Convenient methods for calculating the coefficients $A_{i, k}$ (which naturally depend on the integrand function values encountered) are given in Section 3. A recurrence scheme for calculating the generalized weights $W_{i, k}$ is given in Section 4 (see (4.3) and (4.4)). In Section 5 we discuss empirically the numerical stability and the rate of convergence of our quadrature scheme. In Section 6 we discuss a stopping criterion for the quadrature rule approximation $Q^{\prime}(f)$ given by $\left(\left|A_{l, N-1}\right|+\left|A_{l, N-3}\right|\right)\left|W_{l+1,1}\right|$, which is compared with $\max \left(\left|A_{l, N-1}\right|, 2\left|A_{l, N-3}\right|, 2\left|A_{l, N-s}\right|\right)$ in the $C C$ method. The performance of our stopping criterion is illustrated by numerical examples. In Section 7 we give an algorithm for the closed-type quadrature scheme which is an extension of the CC method. A brief summary and conclusion are given in Section 8 .

2. Uniform Distribution and Chebyshev Distribution. In this section we give the sequence of uniform distribution and the so-called sequence of Chebyshev distribution on the open interval $(-1,1)$ which makes the corresponding sequence of the interpolation polynomials converge uniformly on the closed interval $[-1,1]$ for each 
function analytic on $[-1,1][6$, p. 254]. The projection on the real axis of the uniformly distributed sequence on the unit circle in the complex plane yields the sequence of Chebyshev distribution. Let any positive integer $l$ be written in radix 2 notation as

$$
l=l_{m} l_{m-1} \cdots l_{2} l_{1}=l_{1}+l_{2} \cdot 2+l_{3} \cdot 2^{2}+\cdots+l_{m} \cdot 2^{m-1},
$$

where $m=\left[\log _{2} l\right]+1, l_{i}=0$ or $1(1 \leqslant i \leqslant m-1)$, and $l_{m}=1$.

Lemma 2.1. Define a fraction $\alpha_{l}$ lying between 0 and 1 by

$$
\alpha_{l}=l_{1} \cdot 2^{-1}+l_{2} \cdot 2^{-2}+\cdots+l_{m-1} \cdot 2^{-m+1}+0 \cdot 2^{-m}+1 \cdot 2^{-m-1} .
$$

Then the sequence $\left\{\alpha_{l}\right\}$ is uniformly distributed on $(0,1)$. $l_{m}$.

Note that $l_{m}$ is the coefficient of $2^{-m-1}$, and the coefficient of $2^{-m}$ is zero and not

Proof. Because the sequence $\left\{\alpha_{l}+2^{-m-1}\right\}$ is the so-called Van der Corput sequence [7, p. 127] which is uniformly distributed on $(0,1)$, the sequence $\left\{\alpha_{l}\right\}$ is also uniformly distributed.

Remark. The Van der Corput sequence is known to have a smaller discrepancy than any others that have yet been found [7].

It is easily shown that the sequence $\left\{\alpha_{l}\right\}$ satisfies the recurrence formula (1.1). Table 1 illustrates how the sequence $\left\{\alpha_{l}\right\}$ is constructed from the sequence $\{l\}$.

TABLE 1

Relation between the sequences $\{l\}$ and $\left\{\alpha_{l}\right\}$

\begin{tabular}{rrlc}
$l$ & $l$ & $\alpha_{l}$ & $\alpha_{l}$ \\
\hline 1 & 1 & .01 & $1 / 4$ \\
2 & 10 & .001 & $1 / 8$ \\
3 & 11 & .101 & $5 / 8$ \\
4 & 100 & .0001 & $1 / 16$ \\
5 & 101 & .1001 & $9 / 16$ \\
6 & 110 & .0101 & $5 / 16$ \\
7 & 111 & .1101 & $13 / 16$
\end{tabular}

The values in the second and third columns are in the radix 2 notation.

COROLlaRY. The sequence $\left\{\cos 2 \pi \alpha_{l}\right\}$, whose elements are all distinct, is one of Chebyshev distribution on $(-1,1)$.

The sequence $\left\{\cos 2 \pi\left(\alpha_{l}+2^{-m-1}\right)\right\}$ directly constructed from the Van der Corput sequence is also a sequence of Chebyshev distribution, but we cannot use this sequence as the sample points because some of its elements coincide with each other. Figure 1 illustrates how the points $\left\{\cos 2 \pi \alpha_{l}\right\}$ are arranged on $(-1,1)$. 


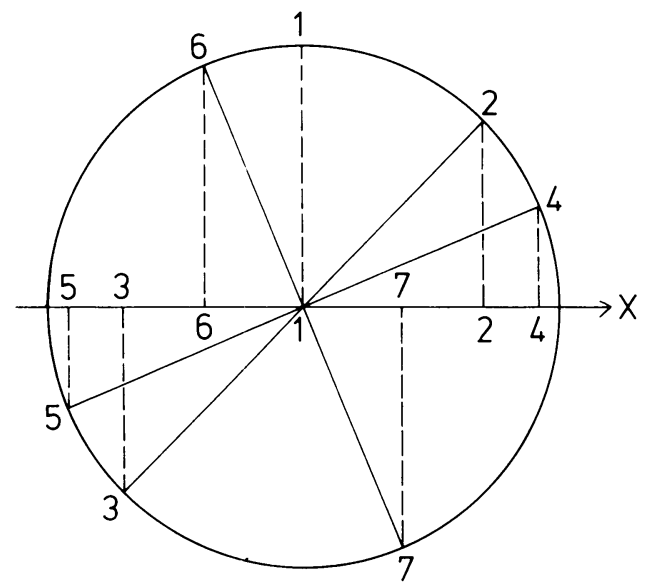

Figure 1

Arrangement of points, $\left\{\cos 2 \pi \alpha_{l}\right\}$, for $l=1,2, \ldots, 7$. Numbers on the unit circle and $x$-axis represent the values of $l$.

LEMMA 2.2. Let $N$ be a power of $2, N=2^{n}$. Then the set of $N$ abscissae

$$
x_{l N+k}=\cos 2 \pi \alpha_{l N+k}, \quad k=0,1, \ldots, N-1,
$$

coincides with the set

$$
\cos 2 \pi\left(\alpha_{l}+j\right) / N, \quad j=0,1, \ldots, N-1 .
$$

Proof. Repeatedly applying the recurrence relation (1.1) gives

$$
\alpha_{l N+k}=\left(\alpha_{l}+k^{*}\right) / N,
$$

where $k^{*}$ denotes the bit reversal of $n$-bit integer $k$. Thus Lemma 2.2 is established.

\section{Corollary.}

$$
2^{N-1} \prod_{k=0}^{N-1}\left(x-x_{l N+k}\right)=T_{N}(x)-\cos 2 \pi \alpha_{l} .
$$

LEMma 2.3. The set of $N-1$ abscissae for $N=2^{n}$,

$$
x_{k}=\cos 2 \pi \alpha_{k}, \quad k=1,2, \ldots, N-1,
$$

coincides with the set

$$
\cos \pi k / N, \quad k=1,2, \ldots, N-1 .
$$

Proof. The proof is by induction. The case $n=1$ is trivial because $\alpha_{1}=\frac{1}{4}$. From (2.3) we have, for the integer $k<2^{n}$,

$$
\cos 2 \pi \alpha_{2^{n}+k}=\cos 2 \pi\left(\alpha_{1}+k^{*}\right) / 2^{n}=\cos \pi\left(\frac{1}{2}+2 k^{*}\right) / 2^{n},
$$


where $1 \leqslant k^{*}<2^{n}$. Using the obvious relation, for $2^{n-1} \leqslant k^{*}<2^{n}$, in (2.5)

$$
\cos \pi\left(\frac{1}{2}+2 k^{*}\right) / 2^{n}=\cos \pi\left(2^{n+1}-2 k^{*}-\frac{1}{2}\right) / 2^{n} \text {, }
$$

we have

$$
\left\{\cos 2 \pi \alpha_{2^{n}+k}\right\}=\left\{\cos \pi\left(k+\frac{1}{2}\right) / 2^{n}\right\} \quad\left(1 \leqslant k<2^{n}\right) .
$$

From the induction hypothesis and (2.6) we have

$$
\begin{aligned}
& \left\{\cos 2 \pi \alpha_{k}\right\}, \quad\left(1 \leqslant k<2^{n+1}\right) \\
& \quad=\left\{\cos \pi k / 2^{n}, \cos \pi / 2^{n+1}, \cos \pi\left(\frac{1}{2}+k\right) / 2^{n}\right\} \quad\left(1 \leqslant k<2^{n}\right) \\
& \quad=\left\{\cos \pi k / 2^{n+1}\right\} \quad\left(1 \leqslant k<2^{n+1}\right) .
\end{aligned}
$$

Thus, the lemma is proved for arbitrary $n$.

Corollary.

$$
2^{N-1} \prod_{k=1}^{N-1}\left(x-\cos 2 \pi \alpha_{k}\right)=2^{N-1} \prod_{k=1}^{N-1}(x-\cos \pi k / N)=U_{N-1}(x) .
$$

From (2.4) and (2.7) we have (1.6).

THEOREM 2.4. Let $l$ be an integer expressed as (2.1) and let $\omega_{l-1}(x)$ denote the polynomial of degree $l-1$ defined by (1.5). Then

$$
\omega_{l-1}(x)=2^{\Sigma l_{\nu}} U_{2^{m} 1_{-1}}(x) \prod_{\left(l_{\nu}=1\right)}\left\{T_{2^{n}} \text { l }(x)+T_{2^{n-1}}\left(\cos 2 \pi \alpha_{l}\right)\right\},
$$

where $\nu$ in the summation and product ranges from 1 to $m-1$, and $T_{n}(x)=\cos n \theta$, $U_{n}(x)=\sin (n+1) \theta / \sin \theta$ with $x=\cos \theta$ are the Chebyshev polynomials of the first and second kinds of degree $n$, respectively.

To prove this theorem, we need Lemma 2.5.

LemMa 2.5. Let $K_{\nu}$ be the set of positive integers defined by

$$
\begin{aligned}
K_{\nu}=\left\{k \mid l_{\mu} \cdot 2^{\mu-1}+\cdots+l_{m} \cdot 2^{m-1}\right. & \leqslant k<l_{\nu} \cdot 2^{\nu-1} \\
& \left.+l_{\mu} \cdot 2^{\mu-1}+\cdots+l_{m} \cdot 2^{m-1}\right\} \quad\left(l_{m}=1\right),
\end{aligned}
$$

where $\mu$ is the integer nearest to $\nu$ such that $l_{\mu} \neq 0, l_{\nu} \neq 0$ and $\mu>\nu$, and

$$
K_{m}=\left\{k \mid 0 \leqslant k<l_{m} \cdot 2^{m-1}\right\} \text {, }
$$

then

$$
2^{2^{\nu-1}} \prod_{k \in K_{\nu}}\left(x-\cos 2 \pi \alpha_{k}\right)=2\left\{T_{2^{\nu-1}}(x)+T_{2^{\nu-1}}^{\prime}\left(\cos 2 \pi \alpha_{l}\right)\right\} .
$$

Proof. For any complex number $z$ we have

$$
\begin{aligned}
& \prod_{k \in K_{\nu}}\left\{z-\exp \left(2 \pi i \alpha_{k}\right)\right\} \\
& =\prod_{0 \leqslant k<2^{\nu-1}}\left[z-\exp \left\{2 \pi i\left(k / 2^{\nu-1}+l_{\mu} \cdot 2^{-\mu}+\cdots+l_{m} \cdot 2^{-m}+2^{-m}\right)\right\}\right] \\
& =z^{2^{\nu-1}}-\exp \left\{2^{\nu} \pi i\left(l_{\mu} \cdot 2^{-\mu}+\cdots+l_{m} \cdot 2^{-m}+2^{-m}\right)\right\} \\
& =z^{2^{\nu-1}}+\exp \left(2^{\nu} \pi i \alpha_{l}\right) .
\end{aligned}
$$


If we set $z=\exp (i \theta)$, the Chebyshev polynomial is expressed as

$$
T_{n}(x)=\left(z^{n}+z^{-n}\right) / 2, \quad x=\left(z+z^{-1}\right) / 2 .
$$

From (2.11) and (2.12) we can easily prove (2.10).

Theorem 2.4 is proved from this lemma and (2.7).

Remark. The generalized weights $W_{i, k}$ in (1.8) are computed recursively by using Theorem 2.4 (see (4.3) and (4.4)).

Corollary.

$$
\max _{0 \leqslant \theta<2 \pi}\left|\omega_{l-1}(\cos \theta) \sin \theta\right| \leqslant 4^{\Sigma l_{\nu}}<l^{2}
$$

where the equality holds only for $l=2^{m-1}$, that is, $\sum l_{\nu}=0$, when we have $\max \left|\omega_{l-1}(\cos \theta) \sin \theta\right|=1$.

The abscissae $\left\{\cos 2 \pi \alpha_{l}\right\}$ are computed recursively as follows. Because $\cos 2 \pi \alpha_{2 l+i}$ $=(-1)^{i} \cos \pi \alpha_{l},(i=0,1)$, only $\left\{\cos \pi \alpha_{l}\right\}$ are required. From (1.1) we have

$$
\begin{aligned}
\alpha_{2^{k}} & =\alpha_{2^{k-1}} / 2, \\
\alpha_{2^{k}+j} & =\alpha_{2^{k-1}+j}-\alpha_{2^{k}} \quad\left(1 \leqslant j<2^{k-1}\right), \\
\alpha_{2^{k+1}-j-1} & =1-\left(\alpha_{2^{k-1}+j}+\alpha_{2^{k}}\right) \quad\left(0 \leqslant j<2^{k-1}\right), k=1,2, \ldots .
\end{aligned}
$$

By using the recurrence relation (2.14) with the starting value $\alpha_{1}=\frac{1}{4}$, the $2^{n}-1$ elements of $\left\{\cos \pi \alpha_{l}\right\} \quad\left(1 \leqslant l<2^{n}\right)$, are evaluated with $n$ square roots and $2^{n}$ multiplications.

3. Interpolation Scheme on the Open Interval $(-1,1)$. We shall make use of the sequence of abscissae $\left\{\cos 2 \pi \alpha_{l}\right\}$ given in the previous section to construct the sequence of interpolation polynomials recursively by adding the fixed number of sample points to the previous one.

3.1. Interpolation Polynomial. Let $N$ be a constant of the form $2^{n}$, and let $p_{l}(x)$ denote the interpolation polynomial for a function $f(x)$ over $[-1,1]$ based on the $(l+1) N-1$ abscissae $\left\{\cos 2 \pi \alpha_{k}\right\}, 1 \leqslant k<(l+1) N$, which prove to be zeros of $U_{N-1}(x) \omega_{l}\left(T_{N}(x)\right)$ from (1.6).

LEMMA 3.1. The polynomial $p_{0}(x)$ is expressed in terms of $U_{k}(x)$,

$$
p_{0}(x)=\sum_{k=1}^{N-1} A_{0, k} U_{k-1}(x)
$$

where

$$
A_{0, k}=\frac{2}{N} \sum_{j=1}^{N-1} f(\cos \pi j / N) \sin \pi j / N \sin \pi j k / N .
$$

Proof. The coefficients $A_{0, k}$ have to satisfy the interpolation condition $p_{0}\left(\cos \theta_{j}^{0}\right)$ $=f\left(\cos \theta_{j}^{0}\right)$, where $\left\{\theta_{j}^{0}\right\}=\left\{2 \pi \alpha_{j}\right\}=\{\pi j / N\},(1 \leqslant j<N)$, that is,

$$
f(\cos \pi j / N) \sin \pi j / N=\sum_{k=1}^{N-1} A_{0, k} \sin \pi j k / N \quad(1 \leqslant j<N) .
$$

By using the orthogonality of the sine function in (3.3) we have (3.2). 
The right-hand side of (3.2) can be computed using the Fast Fourier Transform (FFT).

For the integer $l \geqslant 1$, the polynomial $p_{l}(x)$ is expressed in the form (1.7). Suppose that $p_{l-1}(x)$ is known, that is, all $A_{i, k}(0 \leqslant i<l, 0 \leqslant k<N)$ are given, and we try to evaluate $A_{l, k}(0 \leqslant k<N)$, from which $p_{l}(x)$ is determined. Lemma 2.2 indicates that the $N$ abscissae used in the $l$ th stage are $\cos \theta_{j}^{l},\left(\theta_{j}^{l}=2 \pi\left(\alpha_{l}+j\right) / N, 0 \leqslant\right.$ $j<N)$.

THEOREM 3.2. Let $l$ be the integer expressed in the form (2.1), and define $l^{\prime}=l-$ $2^{m-1}$. Let the formal cosine expansion of $f\left(\cos \theta_{j}^{l}\right) \sin \theta_{j}^{l}$ be

$$
f\left(\cos \theta_{j}^{\prime}\right) \sin \theta_{j}^{l}=\sum_{k=0}^{N-1} a_{k}^{\prime} \cos k \theta_{j}^{l} .
$$

Then the coefficients $A_{l, k},(0 \leqslant k<N)$ in (1.7) are computed recursively as follows.

For each $k$, set the starting value $B_{l}$ as

$$
\begin{aligned}
B_{l^{\prime}}= & a_{k}^{l}-\left(A_{0 . N-k}-A_{0 . k} \cos 2 \pi \alpha_{l}\right) / \sin 2 \pi \alpha_{l} \\
& -\sin 2 \pi \alpha_{l} \sum_{i=1}^{2^{m}} \omega_{i-1}^{1-1}\left(\cos 2 \pi \alpha_{l}\right) A_{i, k}
\end{aligned}
$$

and calculate $B_{l^{\prime}-i}$ by the recurrence relation

$$
\begin{aligned}
& B_{l^{\prime}-i}=\left(B_{l^{\prime}-i+1}-A_{2^{m}++i-1, k}\right) /\left(\cos 2 \pi \alpha_{l}-\cos 2 \pi \alpha_{2^{m}} I_{+i-1}\right) \\
& \left(i=1,2, \ldots, l^{\prime}\right)
\end{aligned}
$$

Then we have $A_{l . h}=B_{0}$, where we have omitted the dependence of $B_{i}$ on $k$ and defined $A_{0,0}=A_{0, N}=0$ for convenience.

Proof. The interpolation condition to determine $A_{l, k}$ is $f\left(\cos \theta_{j}^{\prime}\right)=p_{l}\left(\cos \theta_{j}^{\prime}\right)$; specifically,

$$
\begin{aligned}
f\left(\cos \theta_{j}^{\prime}\right) \sin \theta_{j}^{\prime}= & \sum_{h=0}^{N-1} A_{0, k} \sin k \theta_{j}^{\prime} \\
& +\sin 2 \pi \alpha_{l} \sum_{i=1}^{l} \omega_{i-1}\left(\cos 2 \pi \alpha_{l}\right) \sum_{k=0}^{N-1} A_{i, k} \cos k \theta_{j}^{l} \quad(0 \leqslant j<N) .
\end{aligned}
$$

Substituting the expression (3.4) for $f\left(\cos \theta_{j}^{\prime}\right) \sin \theta_{j}^{\prime}$ inı (3.7) and using the orthogonality of the cosine function, we have the expression for $A_{l, k}$

$$
\begin{aligned}
a_{k}^{l}= & \left(A_{0, N-k}-A_{0, k} \cos 2 \pi \alpha_{l}\right) / \sin 2 \pi \alpha_{l} \\
& +\sin 2 \pi \alpha_{l} \sum_{i=1}^{l} \omega_{i-1}\left(\cos 2 \pi \alpha_{l}\right) A_{i, k} \quad(0 \leqslant k<N) .
\end{aligned}
$$

From (2.2) and (2.8) we have the identity

$$
\sin 2 \pi \alpha_{l} \omega_{2^{m-1}-1}\left(\cos 2 \pi \alpha_{l}\right)=\sin 2 \pi\left(2^{m-1} \alpha_{l}\right)=1 .
$$

Using (3.9) in (3.8) establishes Theorem 3.2.

The right-hand side of (3.5) may be evaluated using Horner's algorithm. The coefficients $a_{k}^{l}$ in (3.4) can be computed by the FFT in the way described later. The numerical stability of the recurrence relations (3.5) and (3.6) is slightly better than 
that of the Newton divided difference formula, because the number of divisions $l+1$ in this formula is reduced to $l^{\prime}+1,\left(l^{\prime} \leqslant l / 2\right)$, in (3.5) and (3.6), although the total sum of the number of divisions and multiplications is not reduced.

By carrying out the procedure given by (3.5) and (3.6) successively for $l=1,2, \ldots$, we obtain the coefficients $A_{l, k}(l=1,2, \ldots)$, which yield the sequence of interpolation polynomials $\left\{p_{l}(x)\right\}$ as given by (1.7).

3.2. Fast Algorithm for Cosine Transform. We describe the algorithm for the fast cosine transform with shift parameter $\alpha$ in order to compute efficiently the discrete Fourier cosine coefficients $a_{k}^{l}$ of (3.4).

Let $t(\theta)$ be the cosine polynomial of degree $N-1$

$$
t(\theta)=\sum_{k=0}^{i-1} a_{k} \cos k \theta .
$$

Then the coefficients $a_{k}$ of $t(\theta)$ are determined by making use of the $N$ function values $t_{j+\alpha}=t(2 \pi(j+\alpha) / N),(0 \leqslant j<N)$, where the shift parameter $\alpha$ is assumed to be a positive fraction. Let us define $A_{k}$ as

$$
A_{k}=\frac{1}{N} \sum_{j=0}^{N-1} t_{j+\alpha} \exp [-2 \pi i k(j+\alpha) / N] \quad(0 \leqslant k<N),
$$

of which the right-hand side can be calculated by the FFT for the real data. Then

$$
\begin{aligned}
A_{N-k} & =\bar{A}_{k} \exp (-2 \pi i \alpha) \quad(0<k<N), \\
A_{N} & =A_{0} \exp (-2 \pi i \alpha),
\end{aligned}
$$

where $\bar{A}_{k}$ denotes the complex conjugate of $A_{k}$. From $(3.10),(3.11),(3.12)$ and the orthogonality of the cosine function with respect to the points $2 \pi(j+\alpha) / N$, it follows that

$$
a_{k}=2 \operatorname{Im}\left(\bar{A}_{N}{ }_{k}\right) / \sin 2 \pi \alpha \quad(0 \leqslant k<N) .
$$

If $t(\theta)$ is the sine polynomial of degree $N$

$$
t(\theta)=\sum_{k}^{N} b_{k} \sin k \theta
$$

then the coefficients $b_{k}$ are given by

$$
\begin{aligned}
& b_{k}=2 \operatorname{Re}\left(A_{N-k}\right) / \sin 2 \pi \alpha \quad(1 \leqslant k<N), \\
& b_{N}=A_{0} / \sin 2 \pi \alpha .
\end{aligned}
$$

4. Open-Type Quadrature Formula. The integration over $[-1,1]$ of the interpolation polynomial $p_{l}(x)$ of degree $(l+1) N-2$ given in the previous section yields the approximation $Q^{\prime}(f)$ given by (1.8) to the integral $Q(f)=\int_{-1}^{1} f(x) d x$. The weights $W_{i, k}$ with $k$ odd in (1.8) are defined by

$$
\begin{aligned}
& W_{0, k}=\int_{-1}^{1} U_{k-1}(x) d x=2 / k, \\
& W_{i, k}=\int_{-1}^{1} U_{N-1}(x) \omega_{i-1}\left(T_{N}(x)\right) T_{k}(x) d x, \quad i \geqslant 1,
\end{aligned}
$$


and $W_{i, k}$ vanishes for $k$ even. From (2.13) we have the bound for $W_{i+1, k}$

$$
\left|W_{i+1, k}\right| \leqslant \int_{0}^{\pi}\left|\sin N \theta \omega_{i}(\cos N \theta) \cos k \theta\right| d \theta<2(i+1)^{2}, \quad i \geqslant 0,
$$

for any $k$.

Now, we shall derive the recurrence relation for $W_{i, k},\left(1 \leqslant i \leqslant 2^{m}\right),(0 \leqslant k<N)$, with $k$ odd. By using the relation (2.8), the weights $W_{2^{n} . k}$ are easily evaluated from (4.1) as

$$
W_{2^{n}, h}=2^{n+1} \cdot N /\left\{\left(2^{n} N\right)^{2}-k^{2}\right\} \quad(n=0,1 \ldots),(k=\text { odd }) .
$$

By making use of (1.5) and (2.8) in (4.1), we have the recurrence relation for $W_{i, k}$

$$
W_{t+2^{\prime \prime} \cdot h}=W_{t, 2^{\prime \prime}{ }^{\prime}+h}+W_{t, 2^{\prime \prime} i_{N h}}-2\left(\cos 2 \pi \alpha_{2 \prime+2 . s}\right) W_{t, h} .
$$

where $t=2^{n}+2^{n-j+1} \cdot s, 0 \leqslant s<2^{\prime}, 1 \leqslant j \leqslant n$ and $n=1,2 \ldots, m-1$. With the starting value $W_{2^{n}, k}$ evaluated by (4.3), $L$ weights $W_{i, k}\left(1 \leqslant i \leqslant 2^{m}, 0 \leqslant k<N, k\right.$ = odd), where $L=2^{m} \cdot N / 2$, can be obtained from the recurrence relation (4.4) with only about $(L / 2) \log _{2} L$ multiplications.

It should be noted that the present method is an extension of the one due to Filippi as the present approximation $Q^{\prime}(f)$ for $l=2^{n}-1$ is identical with that of Filippi [4].

The practical performance of an automatic quadrature scheme depends not only on the number of function evaluations needed to satisfy the required tolerance but also on the overhead in executing the quadrature scheme on a computer.

To reduce this overhead it is desirable that the weights $W_{i, h}$ and the abscissae $\left\{\cos 2 \pi \alpha_{l}\right\}$ should be computed as (4.3), (4.4) and by using the recurrence relation (2.14) once for all, and then be stored in the storage of the computer in advance. From a practical point of view, the increment of the number of sample points $N$ at each step of integration is chosen as $8\left(=2^{3}\right)$.

The results of numerical experiments on the present method suggest that the number of abscissae needed in a double-precision arithmetic (16-place mantissa) implementation in a subroutine library is usually at most $511(=64 \mathrm{~N}-1)$ with $N=8$. From the actual evaluation of the weights $W_{i, 2 k+1}$ we find that the 256 $\left(=2^{6} \cdot 8 / 2\right)$ weights $W_{i .2 h+1}\left(0 \leqslant i<2^{6}\right),(0 \leqslant k \leqslant 3)$, are positive and bounded by $3.6 \cdots$, which is much less than the bound (4.2).

5. Numerical Stability and Rate of Convergence. The numerical stability and rate of convergence in the open-type quadrature scheme is now described.

5.1. Numerical Stability. The open-type formula (1.8) can be rewritten as

$$
Q^{\prime}(f)=\sum_{j=1}^{(l+1) N} w_{j}^{\prime} f\left(x_{j}\right) .
$$

where the weights $w_{j}^{l}$ are given by

$$
w_{j}^{\prime}=\int_{-1}^{1} U_{N-1}(x) \omega_{l}\left(T_{N}(x)\right) /\left\{\left(x-x_{j}\right)\left[U_{N-1}(x) \omega_{l}\left(T_{N}(x)\right)\right]_{x=x_{l}}^{\prime}\right\} d x
$$

and $[\cdots]^{\prime}$ means the derivative with respect to $x[5]$. 
Define a condition number

$$
\sigma^{\prime}=\sum_{j}\left|w_{j}^{\prime}\right| / \sum_{j} w_{j}
$$

Then $\sigma^{\prime} \geqslant 1$. When all weights are positive $\sigma^{\prime}=1$, otherwise $\sigma^{\prime}>1$. The result of numerical evaluation of the weights $w_{j}^{l}$ indicates that with $N=8$ and $l<64$, $\sigma^{\prime} \leqslant 1.5$, except for $l=30$ and $l=62$ in which cases $\sigma^{l}=2.22$ and 3.65 , respectively. (For the closed case $\sigma^{\prime} \leqslant 1.015$.) This fact demonstrates that for practical purposes the present quadrature scheme may be taken to be stable.

It is an open problem to evaluate the best bound of $\sigma^{\prime}$ theoretically.

5.2. Rate of Convergence. Let $\varepsilon_{\rho}$ denote the ellipse in the complex plane $z=x+i y$ with foci $(x, y)=(-1,0),(1,0)$ and semimajor axis $a=\left(\rho+\rho^{-1}\right) / 2$ and semiminor axis $b=\left(\rho-\rho^{-1}\right) / 2$ for a constant $\rho>1$. Furthermore, let $f(z)$ be single-valued and analytic inside and on $\varepsilon_{\rho}$. Then we shall show

$$
E_{l}(f)=Q(f)-Q^{\prime}(f)=O\left(\rho^{-(l+1) N}\right),
$$

for the current case, $N=8,0 \leqslant l<64$.

Achieser [1, p. 219] gives the following lemma.

LEMma 5.1. If $f(z)$ is analytic in $\varepsilon_{\rho}$ and real on $(-1,1)$, then there exists a polynomial $p(x)$ of degree $\leqslant N(l+1)-2$ such that

$$
\max _{-1 \leqslant x \leqslant 1}|f(x)-p(x)| \leqslant \frac{8}{\pi} M(\rho) / \rho^{N(l+1)-1},
$$

where

$$
M(\rho)=\max _{z \in \varepsilon_{\rho}}|\operatorname{Re} f(z)| .
$$

From this lemma and the fact that $E_{l}(p)=0$, it follows that

$$
\begin{aligned}
\left|E_{l}(f)\right| & =\left|E_{l}(f)-E_{l}(p)\right|=\left|E_{l}(f-p)\right| \\
& \leqslant \int_{-1}^{1}|f(x)-p(x)| d x+\sum_{j}\left|w_{j}^{l}\right|\left|f\left(x_{j}\right)-p\left(x_{j}\right)\right| \\
& \leqslant \frac{8 M(\rho)}{\pi}\left[\int_{-1}^{1} d x+\sum_{j}\left|w_{j}\right|\right] / \rho^{N(l+1)} \quad \text { ।, }
\end{aligned}
$$

where $j$ in the summation ranges from 1 to $N(l+1)-1$. Taking into account the numerical results on $\sigma^{\prime}$, we have that the rate of convergence is geometrical for an analytic function on the closed interval $[-1,1]$.

6. Stopping Criterion. The method of adequate error estimation is very important in enhancing the efficiency and reliability of an automatic quadrature scheme. In what follows a practical estimate of truncation error to the open type formula is given.

The truncation error $E_{l}(f)$ of the approximation $Q^{\prime}(f)$ is given by

$$
E_{l}(f)=\int_{-1}^{1} U_{N-1}(x) \omega_{l}\left(T_{N}(x)\right) 2^{1-(l+1) N} f\left[x, x_{1}, \ldots, x_{(l+1) N-1}\right] d x,
$$

where $f\left[x, x_{1}, \ldots, x_{(l+1) N-1}\right]$ denotes the divided difference of order $(l+1) N-1$ [3]. Let $f(z)$ be single-valued and analytic inside and on $\varepsilon_{\rho}$. Then the divided 
difference in (6.1), which is also expressed in terms of contour integral, can be expanded in Chebyshev series

$$
\begin{aligned}
& 2^{1-(l+1) N} f\left[x, x_{1}, \ldots, x_{(l+1) N-1}\right] \\
& \quad=\frac{1}{2 \pi i} \oint_{\varepsilon_{\rho}} f(z) /\left\{(z-x) U_{N-1}(z) \omega_{l}\left(T_{N}(z)\right)\right\} d z \\
& \quad=\sum_{k=0}^{\infty} a_{l+1 . k} T_{k}(x),
\end{aligned}
$$

where the coefficients $a_{l+1, k}$ are given by

$$
a_{l+1, k}=\frac{2}{\pi} \cdot \frac{1}{2 \pi i} \oint_{\varepsilon_{p}} f(z) \tilde{U}_{k}(z) /\left\{U_{N-1}(z) \omega_{l}\left(T_{N}(z)\right)\right\} d z .
$$

The Chebyshev function of the second kind $\tilde{U}_{k}(z)$ is defined by

$$
\tilde{U}_{k}(z)=\int_{-1}^{1} T_{k}(x) /\left\{(z-x) \sqrt{1-x^{2}}\right\} d x=\pi /\left\{\sqrt{z^{2}-1} w^{k}\right\} .
$$

where $w=z+\sqrt{z^{2}-1}$ and $|w|>1$ for $z \notin[-1,1]$. From (6.1), (6.2) and the definition (4.1) of weights we have

$$
E_{l}(f)=\sum_{h=0}^{\infty} a_{l+1.2 h+1} W_{l+1.2 k+1}
$$

Suppose that $f(z)$ is a meromorphic function which has $J$ simple poles at the points $z_{j}(j=1,2, \ldots, J)$ outside $\varepsilon_{\rho}$ with residues Res $f\left(z_{j}\right)$. Then, carrying out the contour integral of $(6.3)$, we have

$$
a_{l+1, k}=-\frac{1}{\pi} \sum_{j=1}^{J} \tilde{U}_{k}\left(z_{j}\right) \operatorname{Res} f\left(z_{j}\right) /\left\{U_{N-1}\left(z_{j}\right) \omega_{l}\left(T_{N}\left(z_{j}\right)\right)\right\} .
$$

From (6.4), (6.6) it can be seen that as $k$ increases $\left|a_{l+1 . k}\right|$ decreases exponentially like $r^{-k}$, where $r=\min ,\left|z_{j}+\sqrt{z_{j}^{2}-1}\right|>1$. Taking into account this fact and the boundedness of $\left|W_{l+1, k}\right|$, (4.2) for fixed $l$, we may estimate the truncation error $E_{l}(f)$ in (6.5) as follows,

$$
\left|E_{l}(f)\right| \lesssim\left|W_{l+1,1}\right| \sum_{k=0}^{\infty}\left|a_{l+1,2 k+1}\right| \simeq\left|W_{l+1,1}\right|\left|a_{l+1,1}\right| /\left(1-r^{-2}\right) .
$$

If we use the relation $\left|a_{l+1,1}\right| \simeq\left|a_{l, N-1}\right| r^{-2} \simeq\left|a_{l, N-3}\right| r^{-4}$, which can be derived by using $\left|T_{N}(z)-\cos 2 \pi \alpha_{l}\right|^{-1} \sim O\left(|w|^{-N}\right)$ in (6.6), then we have

$$
\left|E_{l}(f)\right| \lesssim\left|W_{l+1,1}\right|\left(\left|a_{l, N-1}\right|+\left|a_{l, N-3}\right| r^{-2}\right) /\left(2 r^{2}-2\right) .
$$

When $r^{2} \geqslant 3 / 2$, it is possible to estimate $\left|E_{l}(f)\right|$ by

$$
\left|E_{l}(f)\right| \lesssim\left|W_{l+1,1}\right|\left(\left|a_{l, N-1}\right|+\left|a_{l, N-3}\right|\right) \text {. }
$$


Now we wish to estimate the coefficients of the Chebyshev series of the divided difference (6.2) in terms of the available coefficients $A_{l, k}$, which can be expressed in the form (see Appendix)

$$
\begin{aligned}
A_{l, k}= & \frac{1}{\pi} \cdot \frac{1}{\sin ^{2} 2 \pi \alpha_{l}} \\
& \cdot \frac{1}{2 \pi i} \oint_{\varepsilon_{\rho}}\left\{\tilde{U}_{k}(z)-\left(\cos 2 \pi \alpha_{l}\right) \tilde{U}_{N-k}(z)\right\} \times \frac{\left(w^{N}-\cos 2 \pi \alpha_{l}\right) f(z)}{\left\{U_{N-1}(z) \omega_{l}\left(T_{N}(z)\right)\right\}} d z .
\end{aligned}
$$

Under the same assumption on $f(z)$ as that in the derivation of (6.6), performing the contour integral of (6.8) gives

$$
\begin{aligned}
A_{l, k}= & -\frac{1}{\pi} \cdot \frac{1}{\sin ^{2} 2 \pi \alpha_{l}} \sum_{j=1}^{J}\left\{\tilde{U}_{k}\left(z_{l}\right)-\left(\cos 2 \pi \alpha_{l}\right) \tilde{U}_{N-k}\left(z_{j}\right)\right\} \\
& \times \frac{\left(w_{j}^{N}-\cos 2 \pi \alpha_{l}\right) \operatorname{Res} f\left(z_{l}\right)}{\left\{U_{N-1}\left(z_{j}\right) \omega_{l}\left(T_{N}\left(z_{j}\right)\right)\right\}},
\end{aligned}
$$

where $w_{j}=z_{j}+\sqrt{z_{j}^{2}-1}$. Comparison of (6.6) and (6.9) gives

$$
a_{l+1, k} / A_{l, k}=O\left(r^{-N}\right)
$$

for large $l$, unless the poles $z_{j}$ of $f(z)$ are close to the range $[-1,1]$ on the real axis. On the other hand, since $a_{l+1, k} / a_{l, k}=O\left(r^{-N}\right)$, we have $a_{l, k} \simeq A_{l, k}$. Thus from (6.7) we could estimate the truncation error $\left|E_{l}(f)\right|$ by $e_{l}$ as

$$
e_{l}=\left(\left|A_{l, N-1}\right|+\left|A_{l, N-3}\right|\right)\left|W_{l+1,1}\right|
$$

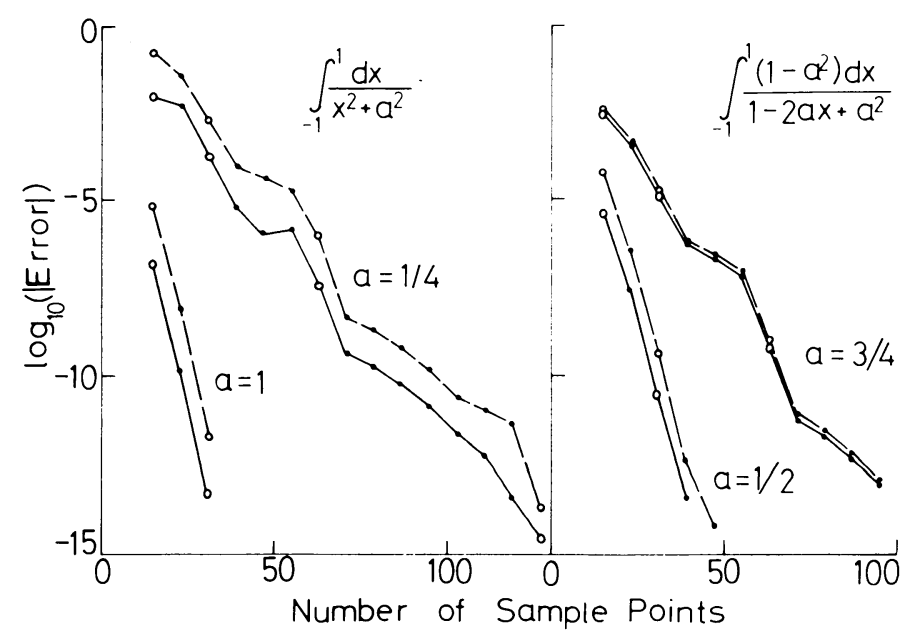

FIGURE 2

Comparison of the true absolute errors and the estimated errors of the quadrature routine applied to the integrals $\int_{-1}^{1} 1 /\left(x^{2}+a^{2}\right) d x$ and $\int_{-1}^{1}\left(1-a^{2}\right) /\left(1-2 a x+a^{2}\right) d x$. Solid curves are the true absolute errors and broken curves are the estimated errors. Circles indicate the errors at the number of sample points $\left(2^{n}-1\right)$ used in the Filippi scheme [4]. 


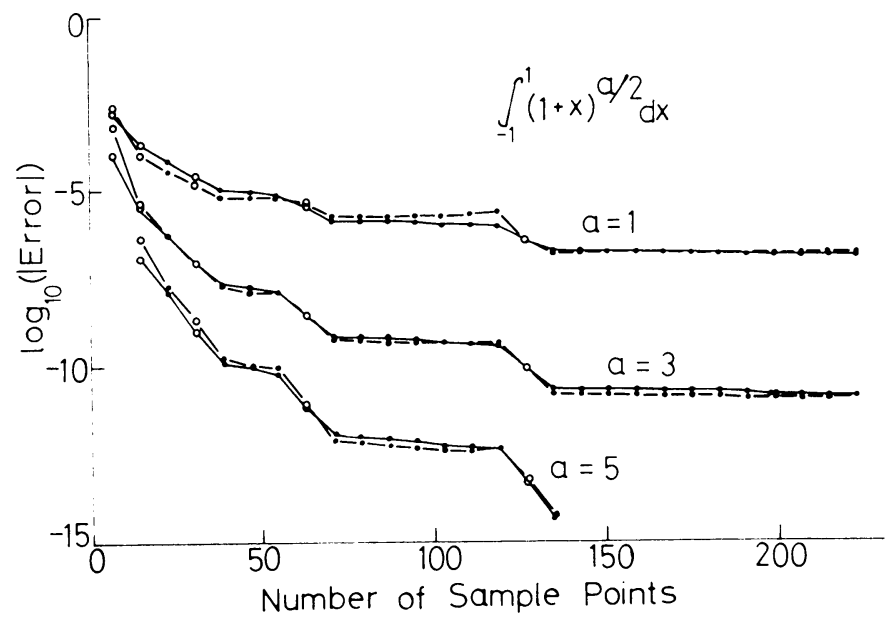

Figure 3

comparison of actual and predicted errors for $!_{-1}^{1}(1+x)^{d 2} d x$.

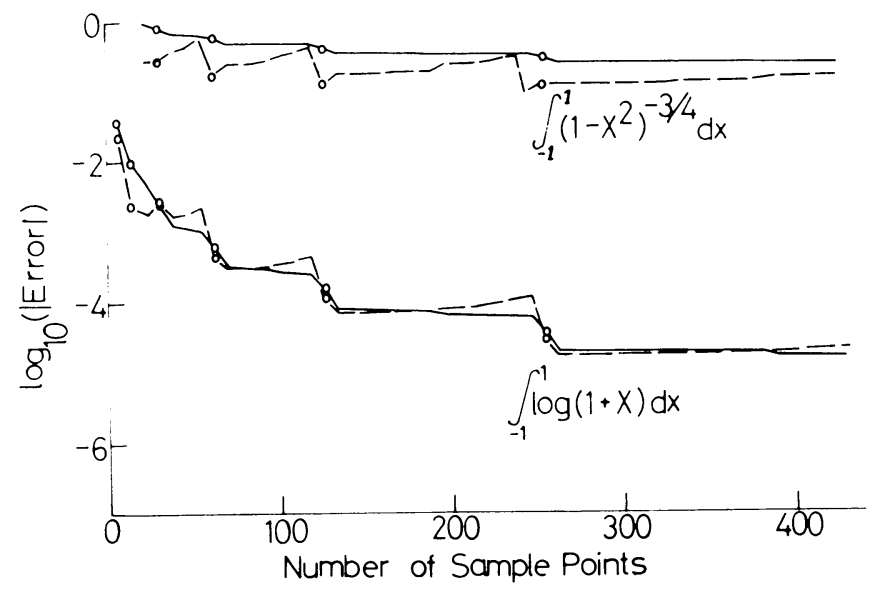

FIGURE 4

Comparison of actual and predicted errors for $\int_{-1}^{1} \log (x+1) d x$ and $\int_{-1}^{1}\left(1-x^{2}\right)^{-3 / 4} d x$.

If some poles $z_{j}$ are very close to the range $[-1,1]$ or the $p$ th derivative $f^{(p)}(x)$, $(p \geqslant 1)$, is discontinuous on $[-1,1]$, the error estimate (6.11) does not hold. To guard against this failure we take the following check procedure. We note that $\left|Q^{2^{n}}(f)-Q^{2^{n-1}}(f)\right|$ should be a better error estimate for tive approximation $Q^{2^{n-1}}(f)$ than $e_{2^{n-1}}$. Therefore, let $\kappa_{2^{n}}^{(1)}$ denote $\left|Q^{2^{n}}(f)-Q^{2^{n-1}}(f)\right| / e_{2^{n-1}}$, and if $\kappa_{2^{n}}^{(1)} \leqslant 1$, then we accept $e_{l}$ defined by (6.11) for $2^{n} \leqslant l<2^{n+1}$. Otherwise, let $\kappa_{2^{n}}^{(2)}$ denote $\kappa_{2^{n}}^{(1)} / \kappa_{2^{n-1}}^{(1)}$, and if $\kappa_{2^{n}}^{(2)} \leqslant 1$, then we take $e_{l} \kappa_{2^{n}}^{(1)}$ as the truncation error estimate. If $\kappa_{2^{n}}^{(1)}>1$ and $\kappa_{2^{n}}^{(2)}>1$, then we take $e_{1} \kappa_{2^{n}}^{(1)} \kappa_{2^{n}}^{(2)}$. 
Figures 2, 3 and 4 illustrate the comparison of the true absolute error and the estimated error by $e_{l}, e_{l} \kappa_{2^{n}}^{(1)}$ or $e_{l} \kappa_{2^{n}}^{(1)} \kappa_{2^{n}}^{(2)}$ for the integral $\int_{-1}^{1} f(x) d x$ where

(1) $f(x)=1 /\left(x^{2}+a^{2}\right), a=1, \frac{1}{4}$,

(2) $f(x)=\left(1-a^{2}\right) /\left(1-2 a x+a^{2}\right), a=\frac{1}{2}, \frac{3}{4}$,

(3) $f(x)=(x+1)^{a / 2}, a=1,3,5$,

(4) $f(x)=\log (x+1)$,

(5) $f(x)=\left(1-x^{2}\right)^{-3 / 4}$,

with $N=8$. Figures 2 and 3 show that the method of the error estimation described above works effectively for well-behaved functions but may occasionally be misleading for integrand functions with discontinuous lower order derivatives on $[-1,1]$.

7. Closed-Type Quadrature Over the Interval $[-1,1]$. In this section we consider an automatic quadrature of closed type which has abscissae on both ends of the interval $[-1,1]$. The procedure for obtaining such a quadrature scheme is similar to that for the open-type one described in the preceding sections.

The sequence of abscissae to be used in this scheme is as follows. The addition of two parameters $\alpha_{-1}=0$ and $\alpha_{0}=\frac{1}{2}$ to the sequence $\left\{\alpha_{i}\right\}(i=1,2 \ldots)$, given in Section 2, yields also a uniformly distributed sequence $\left\{\cos 2 \pi \alpha_{i}\right\}(i=-1,0,1, \ldots)$, on the closed interval $[-1,1]$ whose first $2^{n}+1$ elements coincide with the abscissae $\left\{\cos \pi i / 2^{n}\right\}\left(i=0,1, \ldots, 2^{n}\right)$, that is, zeros of $\left(1-x^{2}\right) U_{2^{n-1}}(x)$, used in the CC method [2].

In a similar way to that in Section 3, let $p_{l}(x)$ denote the interpolation polynomial for a well-behaved function $f(x)$ over $[-1,1]$ based on the $(l+1) N+1$ abscissae $\left\{\cos 2 \pi \alpha_{i}\right\}(-1 \leqslant i<(l+1) N)$, with $N=2^{n}$. Then we have

$$
\begin{aligned}
& p_{l}(x)=p_{l-1}(x)+\left(1-x^{2}\right) U_{N-1}(x) \omega_{l-1}\left(T_{N}(x)\right) \sum_{k=1}^{N} A_{l, k} U_{k-1}(x), \\
& p_{0}(x)=\sum_{k=0}^{N} " A_{0, k} T_{k}(x),
\end{aligned}
$$

where the double prime in the summation denotes a finite sum whose first and last terms are to be halved. The coefficients $A_{0, k}$ in (7.1) are given by

$$
A_{0, k}=\frac{2}{N} \sum_{j=0}^{N}{ }^{\prime \prime} f(\cos \pi j / N) \cos \pi j k / N \quad(0 \leqslant k \leqslant N),
$$

from the interpolation condition that $p_{0}\left(x_{j}\right)=f\left(x_{j}\right)$, where $\left\{x_{j}\right\}=\left\{\cos 2 \pi \alpha_{j}\right\}=$ $\{\cos \pi(j+1) / N\}(-1 \leqslant j<N)$. The interpolation conditions for determining the $N$ coefficients $A_{l, k}(1 \leqslant k \leqslant N)$, in the lth stage of (7.1) are, analogously to (3.7),

$$
\begin{aligned}
f\left(\cos \theta_{j}^{l}\right)= & \sum_{k=0}^{N} " A_{0, k} \cos k \theta_{j}^{l} \\
& +\sin 2 \pi \alpha_{l} \sum_{i=1}^{l} \omega_{i-1}\left(\cos 2 \pi \alpha_{l}\right) \sum_{k=1}^{N} A_{i, k} \sin k \theta_{j}^{l},
\end{aligned}
$$


where $\theta_{j}^{\prime}=2 \pi\left(j+\alpha_{l}\right) / N(0 \leqslant j<N)$. In a similar way to (3.4) the discrete sine transform of the left-hand side of (7.3) yields

$$
a_{k}^{l}=\frac{2}{N} \sum_{j=1}^{N} f\left(\cos \theta_{j}^{\prime}\right) \sin k \theta_{j}^{\prime} \text {. }
$$

In practice this transform $\left\{a_{k}^{l}\right\}(1 \leqslant k \leqslant N)$, is given by (3.15) and computed by making use of FFT (3.11) for real data $f\left(\cos \theta_{j}^{l}\right)$. Consequently, from (7.3) and (7.4) the recurrence formula for $A_{l, k}$ is

$$
a_{k}^{l}=\left(A_{0, k} \cos 2 \pi \alpha_{l}+A_{0 . N-k}\right) / \sin 2 \pi \alpha_{l}+\sin 2 \pi \alpha_{l} \sum_{i=1}^{l} \omega_{i-1}\left(\cos 2 \pi \alpha_{l}\right) A_{i, k},
$$

where we have used the identity

$$
\cos k \theta_{j}^{\prime}=\left\{\cos 2 \pi \alpha_{l} \cdot \sin k \theta_{j}^{\prime}+\sin (N-k) \theta_{j}^{\prime}\right\} / \sin 2 \pi \alpha_{l} .
$$

The first term of the right-hand side of (7.5) must be halved when $k=N$.

The integration of $p_{l}(x)$ expressed by $(7.1)$ over $[-1,1]$ gives the closed-type integration formula

$$
Q^{\prime}(f)=\int_{-1}^{1} p_{l}(x) d x=\sum_{k=0}^{N}{ }^{\prime \prime} A_{0, k} W_{0, k}+\sum_{i=1}^{l} \sum_{k=1}^{N} A_{i, k} W_{i, k} \quad(k=\text { even }),
$$

where the weights $W_{i, k}$ with $k$ even are defined by

$$
\begin{gathered}
W_{0, k}=\int_{-1}^{1} T_{k}(x) d x=2 /\left(1-k^{2}\right), \\
W_{i, k}=\int_{-1}^{1}\left(1-x^{2}\right) U_{N-1}(x) \omega_{i-1}\left(T_{N}(x)\right) U_{k-1}(x) d x, \\
\quad(i=1,2, \ldots),(1 \leqslant k \leqslant N) .
\end{gathered}
$$

These weights may be evaluated by the recurrence relation similar to (4.3) and (4.4). The truncation error $E_{l}(f)$ of the approximation (7.7) could be estimated by the form analogous to (6.11).

The result of numerical evaluation of the condition number $\sigma^{\prime}$ for the closed formula (7.7) indicates that $\sigma^{\prime} \leqslant 1.015$ with $N=8$ and $l<64$, which guarantees, for practical purposes only, the numerical stability of the closed formula.

8. Summary and Concluding Remarks. In this paper we have presented an algorithm to generate the sequence of interpolation polynomials increasing the number of sample points in arithmetic progression. Applying the algorithm, we obtain an automatic quadrature scheme which overcomes the drawback in the Clenshaw-Curtis method that the number of sample points is increased in geometric progression. An adequate method of error estimation is described.

We are grateful to the referee for valuable comments.

Appendix. In this appendix the derivation of (6.8) is given. First we note that the coefficients $A_{l, k}$ in (1.7) can also be regarded as the Fourier Chebyshev coefficients of the divided difference of order $l N-1, f\left[x, x_{1}, \ldots, x_{l N-1}\right]$,

$$
2^{1-l N} f\left[x_{j}, x_{1}, \ldots, x_{l N-1}\right]=\sum_{k=1}^{N-1}{ }^{\prime} A_{l, k} T_{k}\left(x_{j}\right)
$$


where $\left\{x_{j}\right\}=\left\{\cos 2 \pi \alpha_{l N+j}\right\}=\left\{\cos 2 \pi\left(j+\alpha_{l}\right) / N\right\}(0 \leqslant j<N)$. On the other hand, we note that the divided difference is expressed in terms of the contour integral as shown by (6.2). If we set

$$
1 /\left(z-x_{j}\right)=\sum_{k=0}^{N-1} B_{k}(z) T_{k}\left(x_{j}\right) \quad(0 \leqslant j<N) .
$$

where $B_{k}(z)$ is given by

(A.3) $\quad B_{k}(z)=\frac{2}{N} \cdot \frac{1}{\sin ^{2} 2 \pi \alpha_{l}} \sum_{j=0}^{N}\left\{T_{k}\left(x_{j}\right)-\left(\cos 2 \pi \alpha_{l}\right) T_{N-k}\left(x_{j}\right)\right\} /\left(z-x_{j}\right)$,

then from (A.1) and (6.2) we have

$$
A_{l, k}=\frac{1}{2 \pi i} \oint_{\varepsilon_{p}} B_{k}(z) f(z) /\left\{U_{N-1}(z) \omega_{l-1}\left(T_{N}(z)\right)\right\} d z
$$

By making use of the generating function for the Chebyshev polynomial

$$
\frac{1-u x}{1-2 x u+u^{2}}=\sum_{n=0}^{\infty} T_{n}(x) u^{n} \quad(|u|<1),
$$

we obtain the Chebyshev expansion of $1 /(z-x)$ for $z \notin[-1,1]$

$$
1 /(z-x)=2 / \sqrt{z^{2}-1} \sum_{n=0}^{\infty} T_{n}(x) / w^{n}
$$

where $w=z+\sqrt{z^{2}-1}$. From (A.3) and (A.6)

(A.7) $\left(\sin ^{2} 2 \pi \alpha_{l}\right) B_{k}(z)$

$$
\begin{aligned}
& =\frac{4}{N} \sum_{n=0}^{\infty}, \sum_{j=0}^{N-1} T_{n}\left(x_{j}\right)\left\{T_{k}\left(x_{j}\right)-T_{N-k}\left(x_{j}\right) \cos 2 \pi \alpha_{l}\right\} /\left\{w^{n} \sqrt{z^{2}-1}\right\} \\
& =2\left\{\sum_{m=0}^{\infty} \cos \left(2 \pi m \alpha_{l}\right) / w^{m N}\right\}\left\{1 / w^{k}-\left(\cos 2 \pi \alpha_{l}\right) / w^{N-k}\right\} / \sqrt{z^{2}-1} \\
& =\frac{1}{\pi}\left(w^{N}-\cos 2 \pi \alpha_{l}\right)\left\{\tilde{U}_{k}(z)-\tilde{U}_{N-k}(z) \cos 2 \pi \alpha_{l}\right\} /\left\{T_{N}(z)-\cos 2 \pi \alpha_{l}\right\},
\end{aligned}
$$

where again we have used (A.5) and (6.4) in the third equality. Substituting the last expression of (A.7) into (A.4), we have the expression (6.8) for $A_{l, k}$.

Department of Information Science

Faculty of Engineering

Fukui University

Fukui 910, Japan

Department of Information Science

Faculty of Engineering

Nagoya University

Nagoya 464, Japan

Department of Information Science

Faculty of Engineering

Nagoya University

Nagoya 464, Japan 
1. N. I. ACHIESER, Theory of Approximation (translated by C. J. Hyman), Ungar, New York, 1956.

2. C. W. Clenshaw \& A. R. CURTis, "A method for numerical integration on an automatic computer," Numer. Math. v. 2. 1960, pp. 197-205.

3. D. Elliotr, "Truncation errors in two Chebyshev series approximations," Math. Comp.. v. 9. 1965. pp. 234-248.

4. S. FILIPPI, “Angenäherte Tschebyscheff-Approximation einer Stammfunktion-eine Modifikation des Verfahrens von Clenshaw und Curtis." Numer. Math., v. 6, 1964. pp. 320-328.

5. J. P. IмноF, "On the method for numerical integration of Clenshaw and Curtis." Numer. Math., v. 5. 1963, pp. 138-141.

6. V. I. Krylov, Approximate Calculation of Integrals (translated by A. H. Stroud). Macmillan. New York, 1962.

7. L. Kuipers \& H. Niederreiter, Uniform Distribution of Sequences. Wiley. New York. 1974.

8. T. ToriI, T. Hasegawa \& I. Ninomiya. "An automatic integration of interpolatory type increasing the sample points with arithmetic progression," Jooho Shori (Information Processing). v. 19. 1978, pp. 248-255, (in Japanese). 